\title{
Optical load sensors utilizing fiber-Bragg-grating based Fabry-Perot resonators
}

\author{
Xuewen Shu, Kate Sugden, Ian Bennion \\ Photonics Research Group, Aston University, Aston Triangle, Birmingham B4 7ET, United \\ Kingdom \\ Tel: +4401212043549, email:X.Shu@aston.ac.uk
}

\begin{abstract}
We report novel optical load sensors utilizing fiber Bragg grating based Fabry-Perot (FBG-FP) structures. Each transmission peak of a FBG-FP is observed to split into two peaks due to the birefringence effect caused by the transversal loading. Transversal loading can be measured through the wavelength separation of the peak split, which shows a good linear relationship. We also demonstrate a load sensing scheme based on the measurement of the beating frequency of a dualwavelength laser employing a FBG-FP. Such a scheme also provides a new way to generate microwave signals.
\end{abstract}

Keywords: Fiber optics sensors, Fiber Bragg gratings, load sensors, pressure sensors

\section{Introduction}

Fiber-optic transverse load or pressure sensors are of interest for many industrial applications. Such sensors produce minimal disturbance of the measurement environment and are immune to electromagnetic interference. Fiberoptic sensors based on photoinduced fiber Bragg gratings (FBGs) have attracted considerable attention and have been used to measure a variety of physical parameters [1]. Several FBG based schemes for transverse load measurement have also been demonstrated [2-3]. These approaches are generally based on the principle that a load applied to the optical fiber will change its birefringence, therefore, resulting in the reflection spectrum splitting of an FBG in a normal fiber [2] or by changing the spectral separation of the two polarization modes of an FBG in a polarization-maintaining fiber [3]. However, microstrain resolution in load sensing is not easy to achieve using these approaches as the bandwidths of the FBGs are, in general, are too broad in comparison with the splitting of the Bragg reflection peaks. Some approaches aiming at achieving high resolution have also been proposed and demonstrated, these include the use of $\pi$-phase-shifted FBGs [4], long-period fiber gratings [5] and reversible sampled FBGs [6]. In this paper, we report a new high-resolution method for transversal loading measurement, which utilizes fiber Bragg grating based Fabry-Perot (FBG-FP) structures. Transversal loading can be directly measured from the birefringence induced wavelength split of the transmission peaks. A load sensing scheme based on the measurement of the beating frequency of a dual-wavelength laser employing a FBG-FP is also proposed and demonstrated.

\section{Experiment}

Fig.1a schematically illustrates an FBG-FP structure under a loading test, in which an optical fiber containing a FBG-FP and a support fiber were placed in parallel between two smooth metal blocks. The length of the top block was 30 mm, which was the length overwhich the force was applied to the fiber and also fully covered the FBG-FP structures used in our experiments. Fig.1b shows a typical transmission spectrum of an FBG-FP under transversal loading, in which the original transmission spectrum of the FBG-FP without loading is also plotted for comparison. The transmission spectra were measured with a broadband light source and an optical spectrum analyzer (OSA, Ando AQ 6317) with a resolution of $0.01 \mathrm{~nm}$. It is clearly seen in Fig. $1 \mathrm{~b}$ that the number of the sharp transmission peaks for the loaded one was doubled that of the original one. Each transmission peak indeed split into a pair of peaks due to transversal load induced birefringence in the optical fiber. The two peaks for each pair were believed to belong to different polarizations. To verify this, we inserted an in-fiber polarizer and a polarization controller (PC) between the broadband light source and the FBG-FP to control the polarization of the input light. Fig. 2 shows the transmission spectra measured with input light of different polarizations. It is clearly seen that only one peak for each pair (seen with unpolarized light) can be observed when the input light was aligned along $\mathrm{x}$-axis or $\mathrm{y}$-axis of the FBG-FP. For the input polarization of $45^{\circ}$, the two peaks

20th International Conference on Optical Fibre Sensors, edited by Julian Jones, Brian Culshaw,

Wolfgang Ecke, José Miguel López-Higuera, Reinhardt Willsch, Proc. of SPIE Vol. 7503, 750354

(c) 2009 SPIE $\cdot$ CCC code: $0277-786 X / 09 / \$ 18 \cdot$ doi: $10.1117 / 12.834251$

Proc. of SPIE Vol. 7503 750354-1 


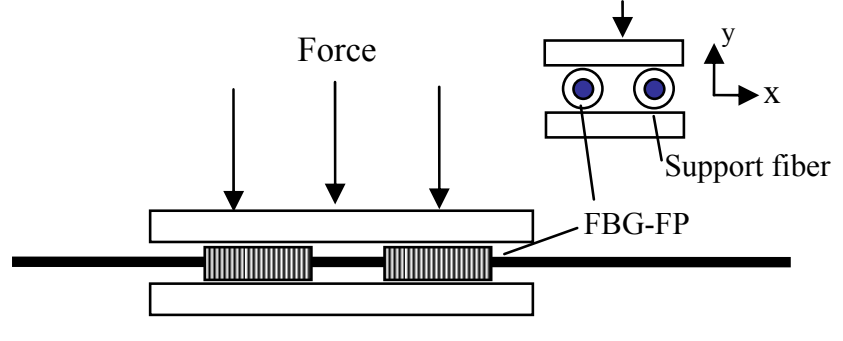

(a)

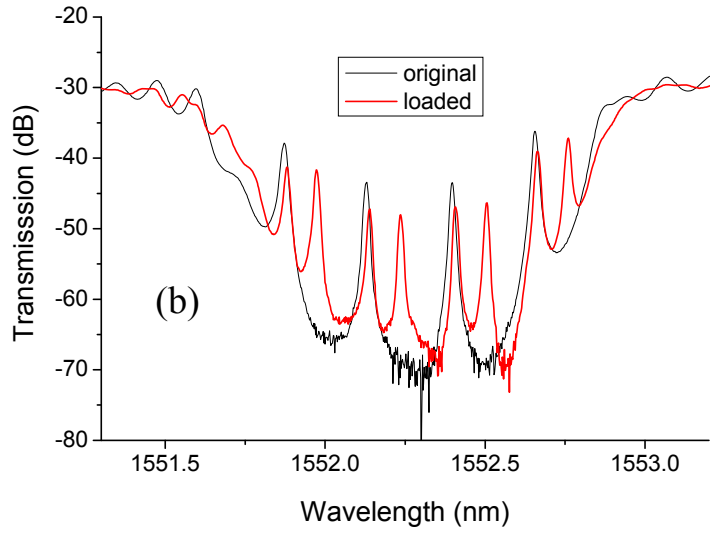

Fig.1 (a) Schematic of the FBG-FP under loading. Inset shows the view from cross section. (b) Transmission spectra of a FBG-FP with/without loading.

can be observed at the same time, which results in a similar spectral profile as the pair measured with unpolarized light. This confirmed that the two peaks originated from two different polarizations.

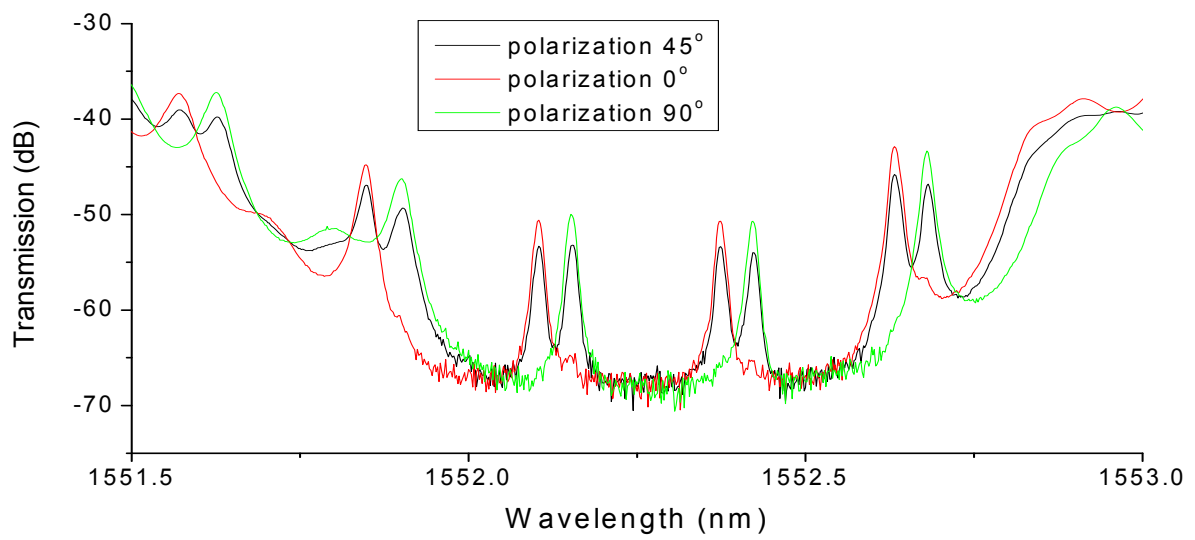

Fig.2 Transmission spectra of a FBG-FP measured with polarized light.

During the load sensing test, we found the separation of the peaks of each pair increased when the transversal load increased. Fig. 3a shows the response of the peak pair of an FBG-FP as a function of the loading. It is seen that one peak (peak2) almost linearly shifted to long wavelength when the load increasing, however, another peak (peak1) almost remained unchanged, which therefore resulted in almost linear variation of their separation with load increasing. It was also observed that the wavelength of peak1 was slightly longer than the original transmission peak of the FBG-FP without loading.

We also found that the wavelength separation of the peak pair strongly depended on the orientation of the transversal load applied. Small, scotch tape flags attached to the fiber were used to indicate the orientation of the fiber. The initial $0^{\circ}$ angle was randomly chosen. Fig. $3 \mathrm{~b}$ shows the response of the FBG-FP as a function of transversal loading with different fiber orientations. It is seen in Fig. $3 b$ that the loading sensitivity for different fiber orientations are significantly different. For orientation $0^{\circ}, 90^{\circ}, 180^{\circ}$ and $270^{\circ}$, the loading sensitivities were found to be $48.9,44.6,74.1$ and $91.8 \mathrm{pm} /\left(\mathrm{N} \mathrm{mm}^{-1}\right)$, respectively. So it is possible for one to obtain different sensitivities and measurement dynamic ranges with one FBG-FP by simply choosing different orientations of the transversal load applied.

FBG-FP structures were also employed in a linear cavity laser to generate dual-wavelength lasing and microwave signals through their beating. Fig.4a shows the schematic of the experimental setup. The cavity of the dual-wavelength 
laser was formed by a loaded FBG-FP with an optical circulator, a polarization controller, a section of $\mathrm{Er} / \mathrm{Yb}$ fiber with length of $1.2 \mathrm{~m}$ and an apodised FBG with a $3 \mathrm{~dB}$ bandwidth of $0.19 \mathrm{~nm}$. The apodised FBG was mounted on a fiber stretcher to adjust central wavelength and therefore can selectively align with a certain transmission peak pair of the loaded FBG-FP. The laser was pumped from a 980/1550 nm WDM coupler spliced at one end of the cavity to minimize the cavity length, and the laser output was launched into a 10:90 coupler $(\mathrm{C}) .10 \%$ of the laser output was monitored by the OSA, while $90 \%$ of the output went into the photo-detector (PD, HP-11982A, 10GHz) after which the beating signal was observed by the electrical spectrum analyzer (ESA, HP 70590A, $40 \mathrm{GHz}$ ). We first tested an FBG-FP with a transmission dip of $\sim 24 \mathrm{~dB}$ and a $3 \mathrm{~dB}$ bandwidth of $\sim 1.8 \mathrm{~nm}$. The dual wavelength lasing was generated and beat each other. Fig. $4 \mathrm{~b}$ shows the beating frequency as a function of the transversal loading. The inset shows the optical spectrum and electrical spectrum of the dual-wavelength laser when the loading was applied at $1.34 \mathrm{~N} / \mathrm{mm}$. It is seen from the
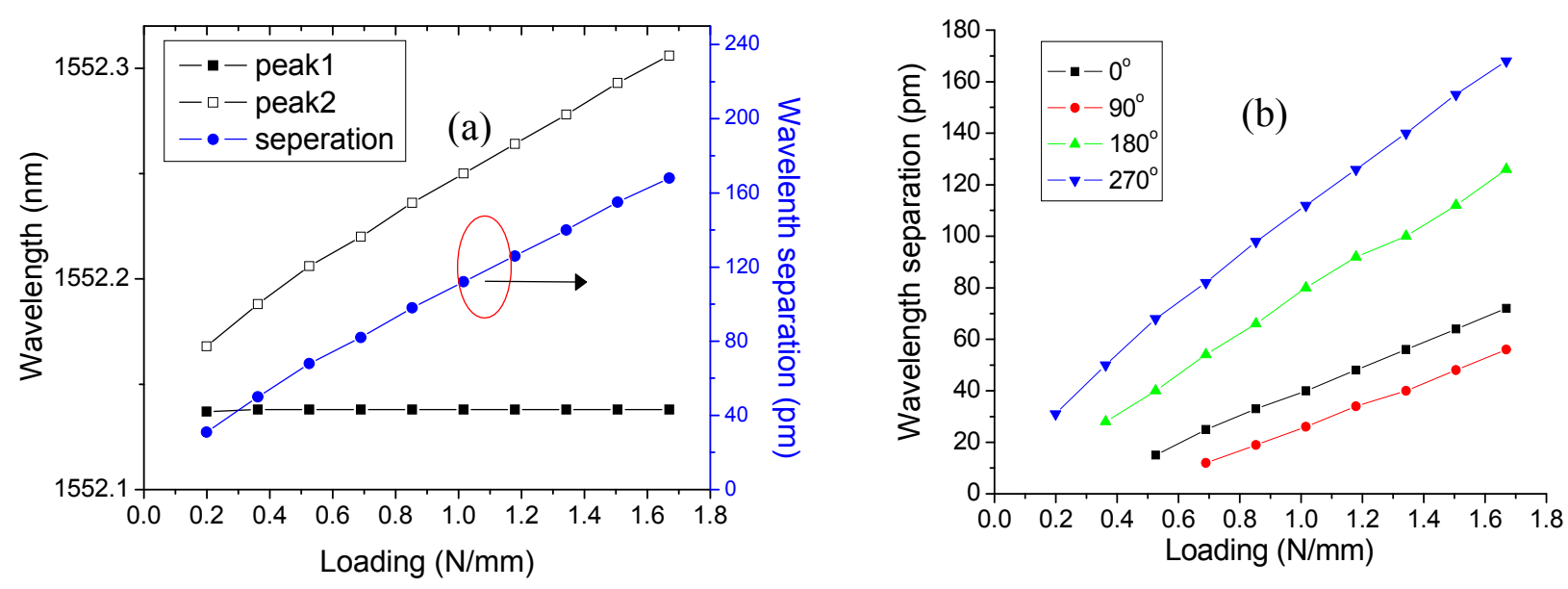

Fig.3 (a) Response of a FBG-FP as a function of transversal loading. (b) Response of a FBG-FP as a function of transversal loading with different fiber orientations.

electrical spectrum that there were multiple beating frequencies, which was due to the fact that the laser operated with multi-longitude modes. The competition of multiple longitudes modes degraded the stability of the laser, however, we can still measure the transversal loading from the center value of the beat frequency. We then tested another FBG-FP with a transmission dip of $\sim 40 \mathrm{~dB}$ and a $3 \mathrm{~dB}$ bandwidth of $1.5 \mathrm{~nm}$. It was found that the stability of the laser was significantly improved by using this stronger FBG-FP since the laser operated at a single longitude mode. The test results are shown in Fig.4c, in which the beating frequency has a very good linear relationship with the transversal loading. It is clearly seen from the electrical spectrum (inset) that the laser had only one beating frequency since it operated with single longitude mode. The loading sensitivity in Fig.4a and $4 \mathrm{~b}$ was found to be 9.71 and $5.95 \mathrm{GHz} / \mathrm{N} / \mathrm{mm}$, respectively.

\section{Conclusion}

We have proposed and demonstrated novel transversal loading sensors utilizing FBG-FP structures. The transversal loading can be measured from the separation of the transmission peak pair of FBG-FP due to transversal loading induced birefringence. We also employ FBG-FP structures in a linear cavity laser to generate dual-wavelength lasing. The transversal loading can also be measured from the beating frequency of the dual-wavelength laser. The proposed transversal loading schemes in this paper have advantages including simplicity, flexibility in choosing sensitivity, high resolution achievable and temperature insensitive.

Acknowledgement: The authors acknowledge the support of the UK EPSRC for this work. 


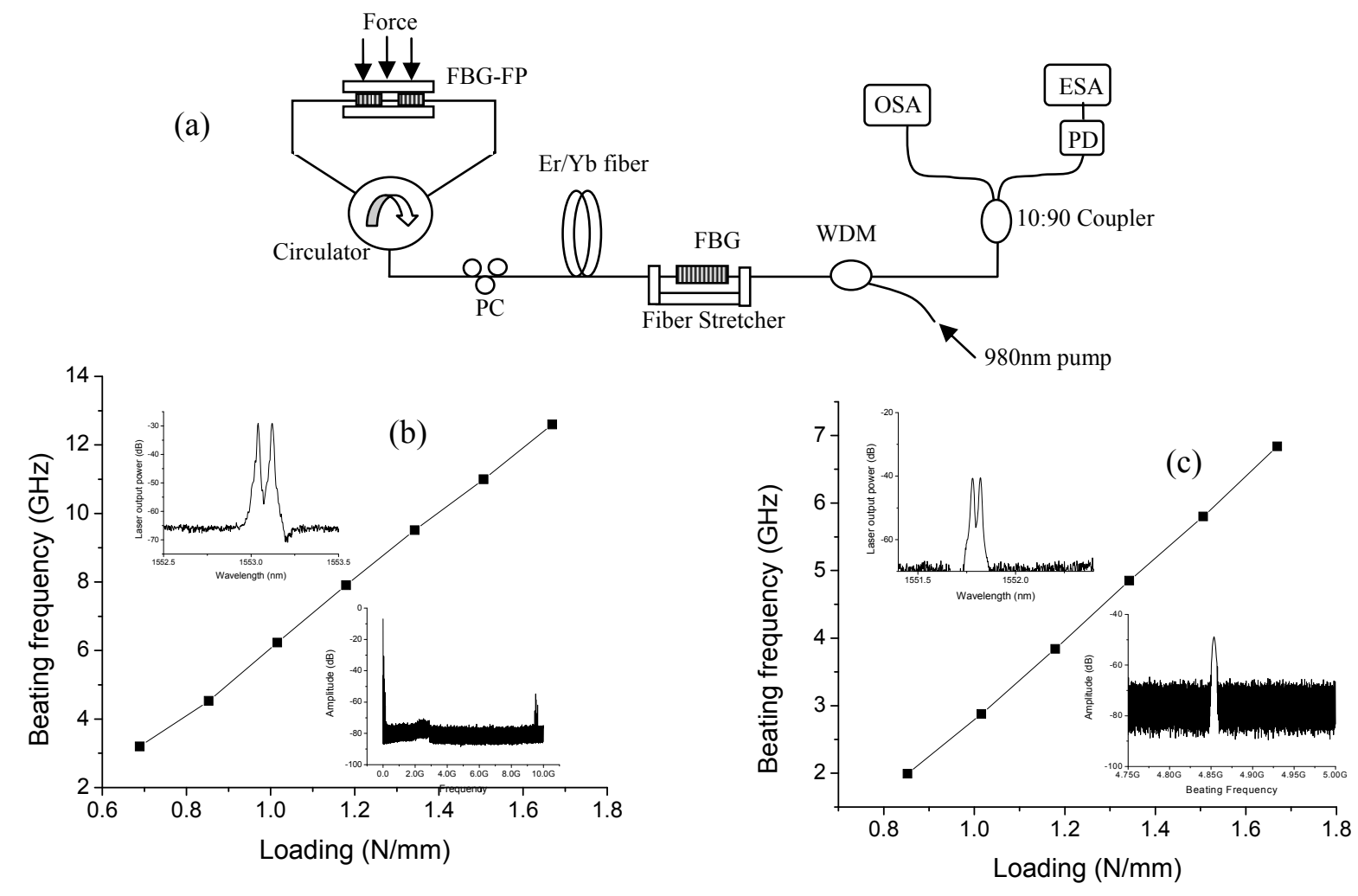

Fig.4 (a) Schematic of the experimental setup for dual-wavelength lasing. (b) Measurement results for a weaker FBG-FP. (c) Measurement results for a stronger FBG-FP

\section{References}

[1] A.D. Kersey, M.A. Davis, H.J. Patrick, M. LeBlanc, K.P. Koo, C.G. Askins, M.A. Putnam, and E.J. Friebele, J. Lightwave Technol., 15,1442 (1997).

[2] R.B. Wagreich, W.A. Atia, H.Singh and J.S. Sirkis, Electon. Lett., 32, 1223 (1996).

[3] C.M. Nelson, D.V. Nelson, and E. Udd, Proc. SPIE 3042, 218 (1997).

[4] M. LeBlanc, S.T. Vohra, T.E. Tsai, and E.J. Friebele, Opt. Lett., 24, 1091 (1999).

[5] Y. Liu, L. Zhang, and I. Bennion, Electon. Lett., 35, 661 (1999).

[6] X. Shu, K. Chisholm, I. Felmeri, K. Sugden, A. Gillooly, L. Zhang, and Ian Bennion, Appl. Phy. Lett., 85, 3003 (2003). 